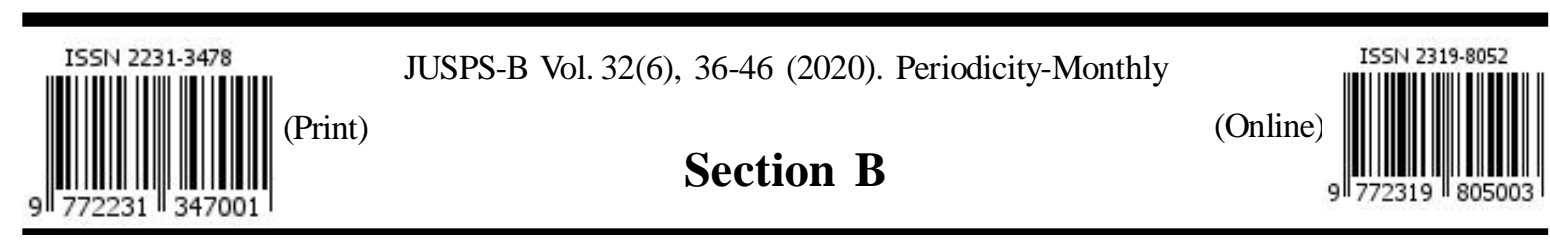

\begin{tabular}{|c|c|}
\hline 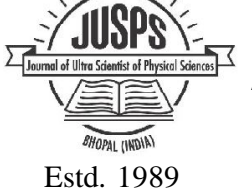 & $\begin{array}{c}\text { JOURNAL OF ULTRA SCIENTIST OF PHYSICAL SCIENCES } \\
\text { An International Open Free Access Peer Reviewed Research Journal of Physical Sciences } \\
\text { website:- } \underline{\text { www.ultrascientist.org }}\end{array}$ \\
\hline
\end{tabular}

\title{
Relativistic Effect of Electrons on Dust Acoustic Solitary waves in unmagnetized plasma
}

\author{
RIJU KUMAR \\ Department of Mathematics, Pandu College, Guwahati-781012, Assam (India) \\ Corresponding Author E-mail : kumarriju1@yahoo.com \\ http://dx.doi.org/10.22147/jusps-B/320601
}

Acceptance Date 24th Novmber, 2020, Online Publication Date 1st December, 2020

\begin{abstract}
In this paper, we study dust acoustic solitary waves (DASW) based on the dust charge $Z_{d}=\frac{n_{d_{0}}}{n_{i_{0}}}=\frac{\text { equilibrium density of dust ions }}{\text { equilibrium density of ions }}$, initial dust speed $U_{d o}$ and initial streaming speed $v_{e o}$ of electrons with the introduction of relativistic effect. Only rarefactive solitons are found to exist in the unmagnetized dusty plasma in presence of relativity for electrons. It is observed that for greater values of dust charge $Z_{d}$ rarefactive solitons exist.
\end{abstract}

Key words : Dust acoustic wave, Dusty plasma, KdV, Soliton.

\section{Introduction}

F ormation of solitary waves in the plasma is being studied both in laboratory and space plasma by many researchers of the world. Recently, the presence of dust grains in the composition of plasma has attracted many workers towards its fold. The presence of dust particles in a plasma influence the plasma properties leading to new significant results.

The dust acoustic waves [DAW] has been investigated by ${ }^{1}$ Rao et.al., for low frequency waves in unmagnetized dusty plasma. On the other hand, dust ion acoustic waves (DIAW) are studied by ${ }^{2} \mathrm{D}^{\prime}$ Angelo, ${ }^{3}$ Nakamura et.al. and ${ }^{4}$ Duan et.al. But existence of DIAW for higher frequency is reported by ${ }^{5}$ Shukla and Silin.

This is an open access article under the CC BY-NC-SA license (https://creativecommons.org/licenses/by-nc-sa/4.0) 
Riju Kumar, JUSPS-B Vol. 32(6), (2020).

In most of the investigations, reductive perturbation method is used. ${ }^{6}$ Duan et.al. have investigated solitary wave in a dusty plasma with variable dust charge through the KdV equation. ${ }^{7}$ Wang and Zhang have studied warm dusty plasmas with two component ions through the KP equation. For two temperature ions, solitons are studied by means of KP equation in dusty plasmas by ${ }^{8}$ Barkan and Javidan. Besides, ${ }^{9}$ Pakzad has studied (DASW) in a dusty plasma with isothermal electrons and nonthermal ions which are detected by Phobas satellite on the upper ionosphere of Mars. Using Sagdeev pseudo-potential method, ${ }^{10} \mathrm{Wang}$ et.al. have shown the existence of solitary waves in dusty plasmas affected by negative ions. Pakzad has reported the existence of only rarefactive solitons at critical temperature in his investigation. ${ }^{11} \mathrm{Z}$. Rahim et.al have studied thes and chaotic motions in Thomas Fermi dusty plasmas. ${ }^{12}$ N.T. Willington et.al have studied the dust acoustic solitary waves in a five component plasma.

In this paper, we consider the existence of dust acoustic solitary waves with weak relativistic effects in electrons in an unmagnetized plasma in presence of negatively charged dust particles. Interestingly, only rarefactive solitons are found to exist in our model of plasma consisting of electrons with weak relativistic effects and non-relativistic massive ion- dust particles.

\section{Basic Equations:}

The governing equations in one- dimension are given by

$$
\begin{aligned}
& \frac{\partial n_{d}}{\partial t}+\frac{\partial}{\partial x}\left(n_{d} u_{d}\right)=0 \\
& \frac{\partial u_{d}}{\partial t}+u_{d} \frac{\partial u_{d}}{\partial x}=Z_{d} \frac{\partial \phi}{\partial x} \\
& \frac{\partial n_{i}}{\partial t}+\frac{\partial}{\partial x}\left(n_{i} v_{i}\right)=0 \\
& \left(\frac{\partial}{\partial t}+v_{i} \frac{\partial}{\partial x}\right)=-Z_{d} \frac{\partial \phi}{\partial x} \\
& \frac{\partial n_{e}}{\partial t}+\frac{\partial}{\partial x}\left(n_{e} v_{e}\right)=0 \\
& \left(\frac{\partial}{\partial t}+v_{e} \frac{\partial}{\partial x}\right)\left(\gamma_{e} v_{e}\right)=\frac{Z_{d}}{Q}\left(\frac{\partial \phi}{\partial x}-\frac{1}{n_{e}} \frac{\partial n_{e}}{\partial x}\right)
\end{aligned}
$$

where $z_{d}$ stands for dust charges, ' $i$ ' for positive ions and ' $e$ ' for electrons respectively, $Q=\frac{m_{e}}{m_{i}}=\frac{\text { mass of the electrons }}{\text { mass of the positive ions }}$ and $Z_{d}=\frac{n_{d_{0}}}{n_{i_{0}}}=\frac{\text { equilibrium density of dust ions }}{\text { equilibrium density of ions }}$

Again for charge imbalances these equations are to be combined by the Poisson equation

$$
\frac{\partial^{2} \phi}{\partial x^{2}}=n_{e}+Z_{d} n_{d}-n_{i}
$$


III. Derivation of the KdV equation :

To derive the KdV equation from the basic equations (1) - (6) for description of the propagation of dust acoustic waves, we expand the variables asymptotically about the equilibrium state in terms of smallness parameter $\varepsilon$ as follows :

$$
\begin{aligned}
& n_{d}=n_{d o}+\varepsilon n_{d 1}+\varepsilon^{2} n_{d 2}+\ldots \\
& n_{i}=n_{i 0}+\varepsilon n_{i 1}+\varepsilon^{2} n_{i 2}+\ldots \\
& n_{e}=1+\varepsilon n_{e 1}+\varepsilon^{2} n_{e 2}+\ldots \\
& u_{d}=u_{d 0}+\varepsilon u_{d 1}+\varepsilon^{2} u_{d 2}+\ldots \\
& v_{i}=\varepsilon v_{i 1}+\varepsilon^{2} v_{i 2}+\ldots \\
& v_{e}=v_{e 0}+\varepsilon v_{e 1}+\varepsilon^{2} v_{e 2}+\ldots \\
& \phi=\varepsilon \phi_{1}+\varepsilon^{2} \phi_{2}+\varepsilon^{3} \phi_{3}+\ldots
\end{aligned}
$$

For unidirectional transformation related to solitary waves in our plasma model, we need to use equation (8) and the stretched variables,

$$
\begin{aligned}
& \xi=\varepsilon^{\frac{1}{2}}(x-U t) \\
& \tau=\varepsilon^{\frac{3}{2}} t
\end{aligned}
$$

in the set of equations (1)-(7). Equating the coefficients of $\varepsilon^{1}$, we get from the first order perturbed quantities, the equation for the phase velocity $U$ namely,

$\frac{1}{Z_{d}-Q\left(U-v_{e_{0}}\right)^{2}\left(1+\frac{3 v_{e_{0}}{ }^{2}}{2 c^{2}}\right)}-\frac{Z_{d} n_{d_{0}}}{\left(U-u_{d_{0}}\right)^{2}}-\frac{n_{i_{0}}}{U^{2}}=0$

This can be solved for the phase velocity $U$ in terms of the equilibrium quantities in it and dust charge $Z_{d}$ and $c$ for relativistic effects.

Again equating the coefficients of $\varepsilon^{2}$ from (1)-(7), we get

$$
\begin{aligned}
& -U \frac{\partial n_{d 2}}{\partial \xi}+\frac{\partial n_{d 1}}{\partial \tau}+\frac{\partial}{\partial \xi}\left(n_{d 0} u_{d 2}+n_{d_{1}} u_{d_{1}}+n_{d 2} u_{d_{0}}\right)=0 \\
& -U \frac{\partial u_{d 2}}{\partial \xi}+\frac{\partial u_{d 1}}{\partial \tau}+u_{d 0} \frac{\partial u_{d 2}}{\partial \xi}+u_{d 1} \frac{\partial u_{d 1}}{\partial \xi}=Z_{d} \frac{\partial \phi_{2}}{\partial \xi}
\end{aligned}
$$


Riju Kumar, JUSPS-B Vol. 32(6), (2020).

$$
\begin{aligned}
& -U \frac{\partial n_{i 2}}{\partial \xi}+\frac{\partial n_{i 1}}{\partial \tau}+\frac{\partial}{\partial \xi}\left(n_{i 0} v_{i 2}+n_{i 1} v_{i 1}\right)=0 \\
& -U \frac{\partial}{\partial \xi} v_{i 2}+\frac{\partial v_{i 1}}{\partial \tau}+v_{i 1} \frac{\partial}{\partial \xi} v_{i 1}=-Z_{d} \frac{\partial \phi_{2}}{\partial \xi} \\
& -U \frac{\partial n_{e 2}}{\partial \xi}+\frac{\partial n_{e 1}}{\partial \tau}+\frac{\partial}{\partial \xi}\left(v_{e 2}+n_{e 1} v_{e 1}+n_{e 2} v_{e 0}\right)=0 \\
& -U \frac{\partial}{\partial \xi}\left(v_{e 2}+\frac{3 v_{e 0}{ }^{2} v_{e 2}}{2 c^{2}}+\frac{3 v_{e 0} v_{e 1}^{2}}{2 c^{2}}\right)+\frac{\partial}{\partial \tau}\left(v_{e 1}+\frac{3 v_{e 0}{ }^{2} v_{e 1}}{2 c^{2}}\right)+ \\
& v_{e 0} \frac{\partial}{\partial \xi}\left(v_{e 2}+\frac{3 v_{e 0}{ }^{2} v_{e 2}}{2 c^{2}}+\frac{3 v_{e 0} v_{e 1}^{2}}{2 c^{2}}\right)+v_{e 1} \frac{\partial}{\partial \xi}\left(v_{e 1}+\frac{3 v_{e 0}{ }^{2} v_{e 1}}{2 c^{2}}\right)=\frac{Z_{d}}{Q}\left(\frac{\partial \phi_{2}}{\partial \xi}-\frac{\partial n_{e 2}}{\partial \xi}+n_{e 1} \frac{\partial n_{e 1}}{\partial \xi}\right) \\
& \frac{\partial^{2} \phi_{1}}{\partial \xi^{2}}=n_{e 2}+Z_{d} n_{d 2}-n_{i 2}
\end{aligned}
$$

Now, eliminating $\frac{\partial u_{d 2}}{\partial \xi}$ from (11) and (12) we get,

$\frac{\partial n_{d 2}}{\partial \xi}+\frac{2 n_{d 0} Z_{d}}{\left(U-u_{d 0}\right)^{3}} \frac{\partial \phi_{1}}{\partial \tau}-\frac{3 n_{d 0} Z_{d}{ }^{2}}{\left(U-u_{d 0}\right)^{4}} \phi_{1} \frac{\partial \phi_{1}}{\partial \xi}+\frac{Z_{d} n_{d 0}}{\left(U-u_{d 0}\right)^{2}} \frac{\partial \phi_{2}}{\partial \xi}=0$

Eliminating $\frac{\partial v_{i 2}}{\partial \xi}$ from (13) and (14) we get,

$$
\begin{aligned}
& \frac{\partial n_{i 2}}{\partial \xi}-\frac{2 n_{i 0} Z_{d}}{\left(U-v_{i 0}\right)^{3}\left(1+\frac{3 v_{i 0}{ }^{2}}{2 c^{2}}\right)} \frac{\partial \phi_{1}}{\partial \tau}-\frac{2 n_{i 0} Z_{d}{ }^{2}}{\left(U-v_{i 0}\right)^{4}\left(1+\frac{3 v_{i 0}{ }^{2}}{2 c^{2}}\right)} \phi_{1} \frac{\partial \phi_{1}}{\partial \xi}- \\
& \frac{n_{i 0} Z_{d}{ }^{2}}{\left(U-v_{i 0}\right)^{4}\left(1+\frac{3 v_{i 0}{ }^{2}}{2 c^{2}}\right)^{2}} \phi_{1} \frac{\partial \phi_{1}}{\partial \xi}+\frac{3 n_{i 0} v_{i 0} Z_{d}{ }^{2}}{c^{2}\left(U-v_{i 0}\right)^{3}\left(1+\frac{3 v_{i 0}{ }^{2}}{2 c^{2}}\right)^{3}} \phi_{1} \frac{\partial \phi_{1}}{\partial \xi}-\frac{n_{i 0} Z_{d}}{\left(U-v_{i 0}\right)^{2}\left(1+\frac{3 v_{i 0}{ }^{2}}{2 c^{2}}\right)^{\frac{\partial \phi_{2}}{\partial \xi}}=0}
\end{aligned}
$$

Further, eliminating $\frac{\partial v_{e 2}}{\partial \xi}$ from (15) and (16) we get, 
$\frac{\partial n_{e 2}}{\partial \xi}+\left[\frac{Z_{d}}{\left(v_{e 0}-U\right)\left\{z_{d}-Q\left(v_{e o}-U\right)^{2}\left(1+\frac{3 v_{e 0}^{2}}{2 c^{2}}\right)\right\}}-\right.$

$\left.\frac{Z_{d}}{\left(v_{e 0}-U\right)\left\{z_{d}-Q\left(v_{e 0}-U\right)^{2}\left(1+\frac{3 v_{e 0}^{2}}{2 c^{2}}\right)\right\}^{2}}-\frac{Q Z_{d}\left(v_{e 0}-U\right)\left(1+\frac{3 v_{e 0}^{2}}{2 c^{2}}\right)}{\left\{z_{d}-Q\left(v_{e 0}-U\right)^{2}\left(1+\frac{3 v_{e 0}^{2}}{2 c^{2}}\right)\right\}^{2}}\right] \frac{\partial \phi_{1}}{\partial \tau}+$

$\left[\frac{Z_{d}{ }^{4}}{2 Q\left(v_{e 0}-U\right)^{2}\left(1+\frac{3 v_{e 0}{ }^{2}}{2 c^{2}}\right)\left\{z_{d}-Q\left(v_{e o}-U\right)^{2}\left(1+\frac{3 v_{e 0}{ }^{2}}{2 c^{2}}\right)\right\}^{3}}+\right.$

$\frac{Z_{d}{ }^{2}}{2 Q\left(v_{e 0}-U\right)^{2}\left(1+\frac{3 v_{e 0}{ }^{2}}{2 c^{2}}\right)\left\{z_{d}-Q\left(v_{e o}-U\right)^{2}\left(1+\frac{3 v_{e 0}{ }^{2}}{2 c^{2}}\right)\right\}}+$

$\frac{3 v_{e 0} Z_{d}{ }^{4}}{2 Q c^{2}\left(v_{e 0}-U\right)\left(1+\frac{3 v_{e 0}{ }^{2}}{2 c^{2}}\right)^{2}\left\{z_{d}-Q\left(v_{e o}-U\right)^{2}\left(1+\frac{3 v_{e 0}{ }^{2}}{2 c^{2}}\right)\right\}^{3}}+$

$\frac{3 v_{e 0} Z_{d}{ }^{2}}{2 Q c^{2}\left(v_{e 0}-U\right)\left(1+\frac{3 v_{e 0}^{2}}{2 c^{2}}\right)^{2}\left\{z_{d}-Q\left(v_{e o}-U\right)^{2}\left(1+\frac{3 v_{e 0}{ }^{2}}{2 c^{2}}\right)\right\}}+$

$\frac{Z_{d}{ }^{3}}{\left\{z_{d}-Q\left(v_{e o}-U\right)^{2}\left(1+\frac{3 v_{e 0}{ }^{2}}{2 c^{2}}\right)\right\}^{3}}$ 
Riju Kumar, JUSPS-B Vol. 32(6), (2020).

$$
\begin{aligned}
& \frac{Z_{d}{ }^{2}}{\left\{z_{d}-Q\left(v_{e o}-U\right)^{2}\left(1+\frac{3 v_{e 0}{ }^{2}}{2 c^{2}}\right)\right\}^{2}}- \\
& \frac{3 v_{e 0} Z_{d}^{3}}{Q c^{2}\left(v_{e 0}-U\right)\left(1+\frac{3 v_{e 0}{ }^{2}}{2 c^{2}}\right)\left\{z_{d}-Q\left(v_{e o}-U\right)^{2}\left(1+\frac{3 v_{e 0}{ }^{2}}{2 c^{2}}\right)\right\}^{2}}- \\
& Q\left(v_{e 0}-U\right)^{2}\left(1+\frac{3 v_{e 0}{ }^{2}}{2 c^{2}}\right)\left\{z_{d}-Q\left(v_{e o}-U\right)^{2}\left(1+\frac{3 v_{e 0}{ }^{2}}{2 c^{2}}\right)\right\}^{2}- \\
& \left.\frac{Z_{d}{ }^{3}}{2\left\{z_{d}-Q\left(v_{e o}-U\right)^{2}\left(1+\frac{3 v_{e 0}{ }^{2}}{2 c^{2}}\right)\right\}^{3}}\right] \frac{\partial \phi_{1}{ }^{2}}{\partial \xi}-\left(\frac{\left.z_{d}-Q\left(v_{e o}-U\right)^{2}\left(1+\frac{3 v_{e 0}{ }^{2}}{2 c^{2}}\right)\right\}}{\partial \xi} \frac{\partial \phi_{2}}{\partial \xi}\right.
\end{aligned}
$$

Subtracting (19) from (20) and then adding $Z_{d} \times(18)$, we can get with the use of (17)

$$
\frac{\partial \phi_{1}}{\partial \tau}+p \phi_{1} \frac{\partial \phi_{1}}{\partial \xi}+q \frac{\partial^{3} \phi_{1}}{\partial \xi^{3}}=0
$$

where $p=\frac{B}{A}$ and $q=\frac{1}{A}$ with $A$ and $B$ given by

$$
\begin{gathered}
A=\left[\frac{\left(Z_{d}-1\right)}{\left(v_{e 0}-U\right)\left\{z_{d}-Q\left(v_{e o}-U\right)^{2}\left(1+\frac{3 v_{e 0}^{2}}{2 c^{2}}\right)\right\}}-\frac{\left(Z_{d}-1\right)}{\left(v_{e 0}-U\right)\left\{z_{d}-Q\left(v_{e o}-U\right)^{2}\left(1+\frac{3 v_{e 0}^{2}}{2 c^{2}}\right)\right\}^{2}}-\right. \\
\left.\qquad \frac{Q Z_{d}\left(v_{e 0}-U\right)\left(1+\frac{3 v_{e 0}^{2}}{2 c^{2}}\right)}{\left\{Z_{d}-Q\left(v_{e 0}-U\right)^{2}\left(1+\frac{3 v_{e 0}^{2}}{2 c^{2}}\right)\right\}^{2}}+\frac{2 Z_{d}}{U^{3}}+\frac{2 Z_{d}}{\left(U-u_{d 0}\right)^{3}}\right]
\end{gathered}
$$




$$
\begin{aligned}
& B=\left[\frac{\left(Z_{d}^{4}-Z_{d}^{3}\right)}{2 Q\left(v_{e 0}-U\right)^{2}\left(1+\frac{3 v_{e 0}^{2}}{2 c^{2}}\right)\left\{Z_{d}-Q\left(v_{e o}-U\right)^{2}\left(1+\frac{3 v_{e 0}{ }^{2}}{2 c^{2}}\right)\right\}^{3}}+\right. \\
& \frac{\left(Z_{d}{ }^{2}-Z_{d}\right)}{2 Q\left(v_{e 0}-U\right)^{2}\left(1+\frac{3 v_{e 0}^{2}}{2 c^{2}}\right)\left\{Z_{d}-Q\left(v_{e o}-U\right)^{2}\left(1+\frac{3 v_{e 0}^{2}}{2 c^{2}}\right)\right\}}+ \\
& \frac{3 v_{e 0}\left(Z_{d}{ }^{4}-Z_{d}{ }^{3}\right)}{2 Q c^{2}\left(v_{e 0}-U\right)\left(1+\frac{3 v_{e 0}{ }^{2}}{2 c^{2}}\right)^{2}\left\{Z_{d}-Q\left(v_{e o}-U\right)^{2}\left(1+\frac{3 v_{e 0}{ }^{2}}{2 c^{2}}\right)\right\}^{3}}+ \\
& \frac{3 v_{e 0}\left(Z_{d}{ }^{2}-Z_{d}\right)}{2 Q c^{2}\left(v_{e 0}-U\right)\left(1+\frac{3 v_{e 0}^{2}}{2 c^{2}}\right)^{2}\left\{Z_{d}-Q\left(v_{e o}-U\right)^{2}\left(1+\frac{3 v_{e 0}^{2}}{2 c^{2}}\right)\right\}}+ \\
& \frac{\left(Z_{d}{ }^{3}-Z_{d}{ }^{2}\right)}{\left\{z_{d}-Q\left(v_{e o}-U\right)^{2}\left(1+\frac{3 v_{e 0}{ }^{2}}{2 c^{2}}\right)\right\}^{3}}-\frac{\left(Z_{d}{ }^{2}-Z_{d}\right)}{\left\{z_{d}-Q\left(v_{e o}-U\right)^{2}\left(1+\frac{3 v_{e 0}{ }^{2}}{2 c^{2}}\right)\right\}^{2}}- \\
& \frac{3 v_{e 0}\left(Z_{d}{ }^{3}-Z_{d}{ }^{2}\right)}{Q c^{2}\left(v_{e 0}-U\right)\left(1+\frac{3 v_{e 0}{ }^{2}}{2 c^{2}}\right)\left\{Z_{d}-Q\left(v_{e o}-U\right)^{2}\left(1+\frac{3 v_{e 0}{ }^{2}}{2 c^{2}}\right)\right\}^{2}}- \\
& \frac{\left(Z_{d}{ }^{3}-Z_{d}{ }^{2}\right)}{Q\left(v_{e 0}-U\right)^{2}\left(1+\frac{3 v_{e 0}{ }^{2}}{2 c^{2}}\right)\left\{Z_{d}-Q\left(v_{e o}-U\right)^{2}\left(1+\frac{3 v_{e 0}{ }^{2}}{2 c^{2}}\right)\right\}^{2}}-\frac{\left(Z_{d}{ }^{3}-Z_{d}{ }^{2}\right)}{2\left\{Z_{d}-Q\left(v_{e o}-U\right)^{2}\left(1+\frac{3 v_{e 0}{ }^{2}}{2 c^{2}}\right)\right\}^{3}}+ \\
& \left.\frac{3 Z_{d}{ }^{2}}{U^{4}}-\frac{3 Z_{d}{ }^{2}}{\left(U-u_{d 0}\right)^{4}}\right]
\end{aligned}
$$

The KdV equation (21) will give the soliton solution for real suitable values of $p$ and $q$ based on the 
Riju Kumar, JUSPS-B Vol. 32(6), (2020).

parameters involved in (22) and (23). For the desired soliton solution, we consider the transformation $\eta=\xi-V \tau$.

Using the transformation $\eta=\xi-V \tau$, for solitary wave solution, we integrate once and use the boundary conditions $\phi_{1}=0, \frac{d^{2} \phi_{1}}{d \eta^{2}}=0$ as $\eta \rightarrow \infty$ to get

$$
\frac{d^{2} \phi_{1}}{d \eta^{2}}=\left(\frac{V}{q}\right) \phi_{1}-\left(\frac{p}{2 q}\right) \phi_{1}^{2}
$$

Multiplying both sides by $2 \frac{d \phi_{1}}{d \eta}$ and again integrating we get

$$
\frac{d \phi_{1}}{d \eta}= \pm \sqrt{\left(\frac{V}{q}\right)} \phi_{1} \sqrt{\left\{\left(1-\frac{p}{3 V} \phi_{1}\right)\right\}}
$$

Integrating again we get the solitary wave solution

$$
\phi_{1}=\left(\frac{3 V}{p}\right) \operatorname{sech}^{2}\left(\frac{1}{2} \sqrt{\frac{V}{q}} \eta\right)
$$

where $V$ is the velocity with which the solitary waves travel to the right. The corresponding amplitudes $\left(\phi_{0}\right)$ and width $(\Delta)$ of the soliton are respectively $\phi_{0}=\left(\frac{3 V}{p}\right)$ and $\Delta=2 \sqrt{\frac{q}{V}}$

\section{Discussion}

The small amplitudes of the dust acoustic rarefactive solitons are found to increase rapidly to maximum [fig 1(a)] against every critical $Z_{d}$ for small dust charge $Z_{d}>10$ in its narrow lower range and decrease concavely as $Z_{d}$ increases under relativistic effect of electrons. The corresponding width ( $\Delta$ ) [fig 1(b)] of small magnitudes decay convexly and parabolically with $Z_{d}$. The initial dust streaming $u_{d_{0}}$ is found to be responsible to generate dust acoustic rarefactive solitons but of relatively high amplitudes [fig 2(a)]. The amplitudes increase in the initial ranges of $u_{d_{0}}$ upto a critical $u_{d_{0}}$ but decreases at the increase of $u_{d_{0}}$. The corresponding width $(\Delta)$ [fig 2(b)] increases in a regular manner with $u_{d_{0}}$. The amplitudes of rarefactive dust acoustic solitary wave diminishes sharply [fig 3(a)] to nearly zero with range of $v_{e_{0}}$. Besides the width $(\Delta)$ grow in quite opposite (convexly) manner [fig 3(b)] with $v_{e_{0}}$. 


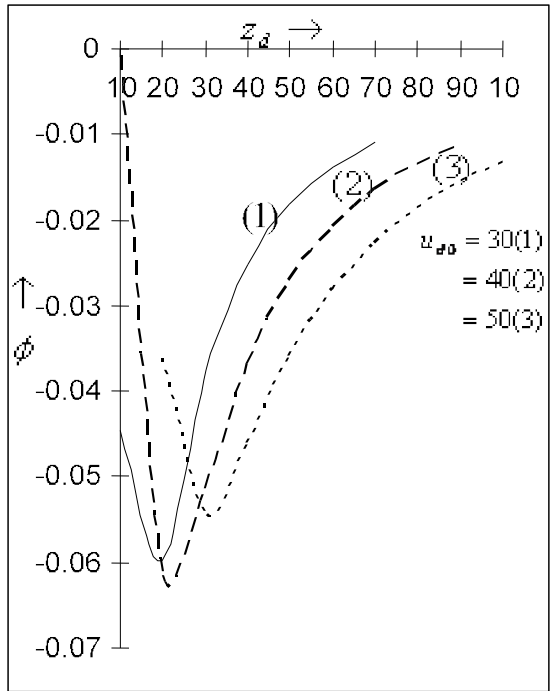

Figure 1(a):

Amplitude $(\varphi)$ of dust acoustic rarefactive solitons versus dust ch arg e $Z_{d}$ for fixed

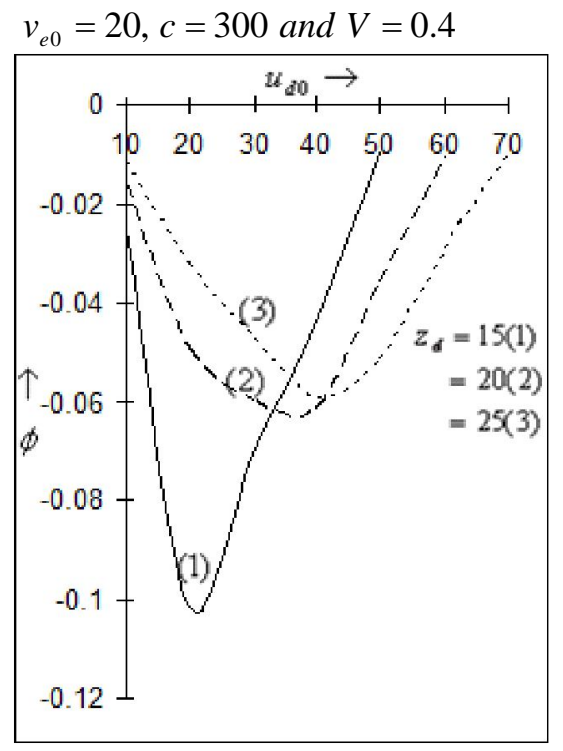

Figure 2(a) :

Amplitude $(\varphi)$ of dust acoustic rarefactive solitons versus initialdust speed $\mathrm{u}_{\mathrm{d} 0}$ for fixed

$v_{e 0}=20, c=300$ and $V=0.4$

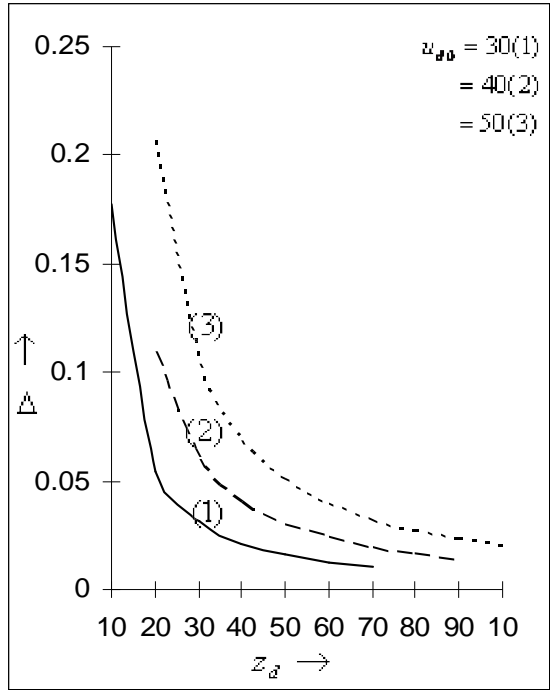

Figure $1(b)$ :

Width $(\Delta)$ of dust acoustic rarefactive solitons versus dust ch arg e $Z_{d}$ for fixed

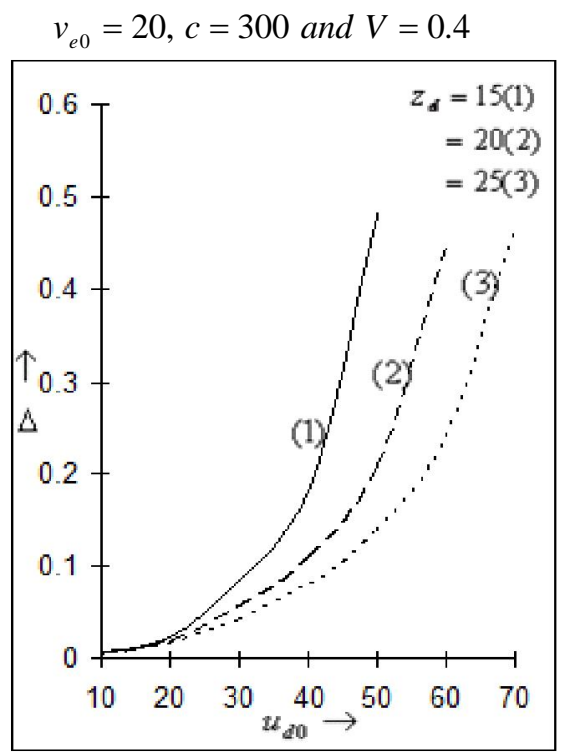

Figure 2(b) :

Width $(\Delta)$ of dust acoustic rarefactive solitons versus initial dust speed $\mathrm{u}_{\mathrm{d} 0}$ for fixed

$v_{e 0}=20, c=300$ and $V=0.4$ 


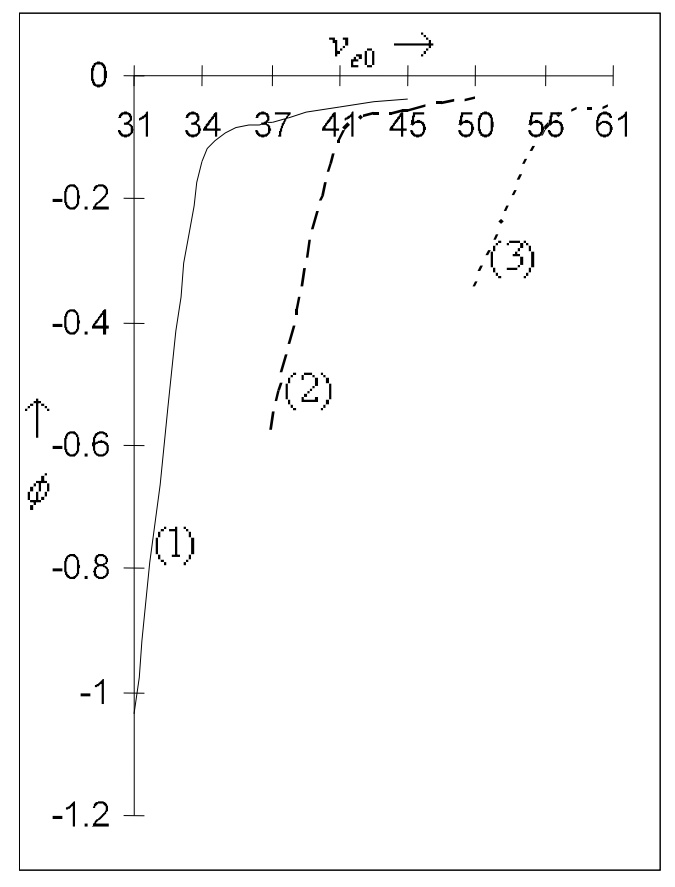

Figure 3(a) :

Amplitude $(\varphi)$ of dust acoustic rarefactive solitons versus initial electron - strea ming $\mathrm{v}_{\mathrm{e} 0}$ for fixed $\mathrm{Z}_{\mathrm{d}}=10, \mathrm{u}_{\mathrm{d} 0}=20, \mathrm{c}=300$, $\mathrm{V}=0.15$

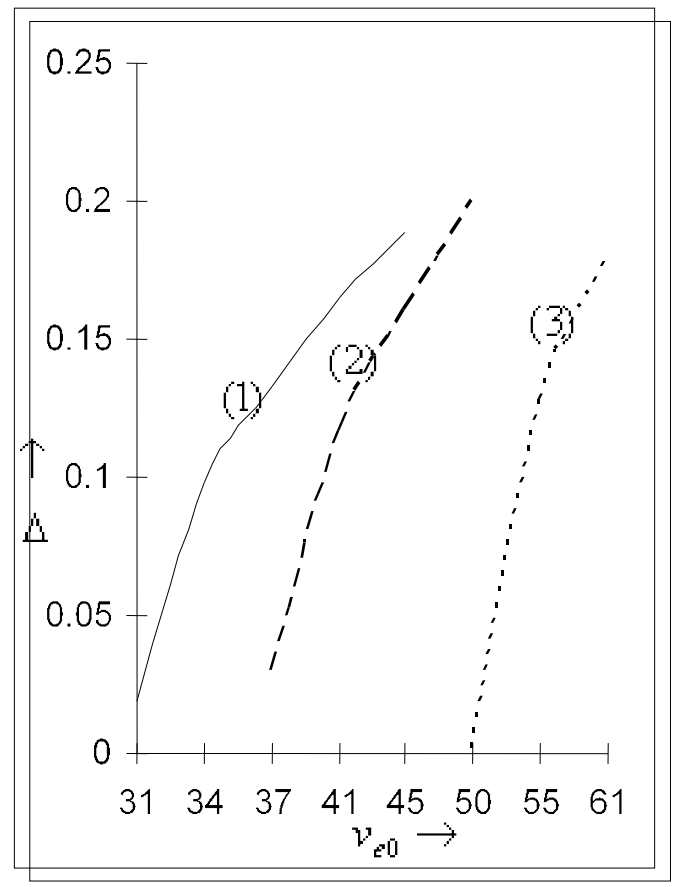

Figure 3(b) :

Width $(\Delta)$ of dust acoustic rarefactive solitons versus initial electron - strea ming $\mathrm{v}_{\mathrm{e} 0}$ for fixed $\mathrm{Z}_{\mathrm{d}}=10, \mathrm{u}_{\mathrm{d} 0}=20, \mathrm{c}=300$, $\mathrm{V}=0.15$

\section{Conclusion}

In this model of dusty plasma, with the introduction of weak relativistic effects in electrons, only rarefactive solitons are established based on the dust charge $Z_{d}$ and the initial streaming speeds $v_{e_{0}}$ of the electrons. For all values of the dust charge $Z_{d}>1$, the soliton is found to be rarefactive. In all cases of this consideration for relativistic situation, we consider $\mathrm{c}=300$ (normalized).

\section{Application and future scope :}

The presence of dust particles in plasmas of space environment has created interest in the domain of plasma research. The role and theoretical applications of dust particles observed in earth's magnetosphere, cometary tail, planetary rings, lower ionosphere of the earth. The usual characteristic plasma phenomena like solitary waves, double layers etc. and their stabilities are being studied with the introduction of dust particles in the plasma. The Vega and Giotto space probes are found to report low frequency noise enhancement in the dusty regions of Halley's comet. A small amount of dust grains of micrometre or sub-micrometre size can be 
considered as charged particles for field emission, ultra-violet ray irradiation in the plasma. The behavioral role of fragmentization of dust grains in the dynamics of plasma primarily can be investigated. Waves and its propagation can be studied explicitly in many branches of science using set of non linear equations. The damping problem in curved beam system can be studied.

\title{
Acknowledgement
}

The author declare no competing financial interest.

\author{
Abbreviation: \\ DAW $\rightarrow$ Dust acoustic wave \\ $\mathbf{K d V} \rightarrow$ Korteweg de Vries
}

\section{References}

1. N.N. Rao., P.K. Shukla. and M.Y. Yu., Planet Space Science. 38, 543-546 (1990).

2. N. D' Angelo., A. Barkan., R.L. Merlino., Phys.Plasmas 2, 3563-3565 (1995).

3. Y. Nakamura., H. Bailung., P.K. Shukla., Phy. Rev. Lett. 83, 1602-1605 (1999).

4. W.S. Duan., K.P.Lu., J.B. Zhao., Chain. Phys. Lett. 18, 1088-1089 (2001).

5. P.K. Shukla., V.P. Sili, Phys. Scr. 45, 508 (1992).

6. W.S. Duan., X.R. Hong., Y.R. Shi, J.A. Sun, Chaos Soliton. Fract. 16, 767-777 (2003).

7. Wang Y.Y., Zhang, J.F., Phys. Lett., A352 (2006).

8. H.R. Barkan., K. Javidian., Chaos Soliton. Fract. (2009, in Press)

9. H.R. Pakzad., Astrophys Space Science. 324, 41 (2009).

10. AG Kharapak, SA Kharapak 25, 034502 (2018)

10. Z.X. Wang, J.Y, Liu et.al., Phys. Lett. A399 (2005a), 96

Z.X. Wang, J.Y, Liu et.al, Phys. Lett. A399 (2005b), 264

11. Z. Rahim, A. Saha et.al., POP25, 0837006(2018)

12. N.T. Willington, Sijo Sebastian, Chandu Venugopal, Research and Reviews, Journal Of Physics 8, 44-51 (2019). 